\title{
Peripheral Administration of NMU Promotes White Adipose Tissue Beiging and Improves Glucose Tolerance
}

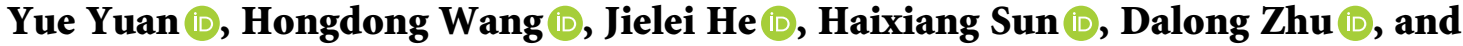 \\ Yan Bi \\ Department of Endocrinology, Affiliated Drum Tower Hospital, Medical School of Nanjing University, Nanjing, China \\ Correspondence should be addressed to Yan Bi; biyan@nju.edu.cn
}

Received 20 May 2021; Revised 25 June 2021; Accepted 21 July 2021; Published 2 August 2021

Academic Editor: Muhittin Yurekli

Copyright (C) 2021 Yue Yuan et al. This is an open access article distributed under the Creative Commons Attribution License, which permits unrestricted use, distribution, and reproduction in any medium, provided the original work is properly cited.

\begin{abstract}
Purpose. Targeting white adipose tissue (WAT) beiging has been proposed as an effective way to increase thermogenesis and improve glucose metabolism. Neuromedin U (NMU) is a neuropeptide that could increase energy expenditure, while its effects on WAT beiging and glucose homeostasis remain to be investigated. Methods. Male C57BL/6 mice were fed with high fat diet (HFD) to induce obesity and hyperglycemia and then treated with chronic subcutaneous injection of NMU. Body weight and food intake were recorded daily. After 14 days of injection, intraperitoneal glucose tolerance tests and 18F-fluorodeoxyglucose micro-positron emission tomography/computed tomography (18F-FDG micro-PET/CT) scans were conducted. Subcutaneous WAT (sWAT) and interscapular brown adipose tissue were collected for the evaluation of adipocyte size, expression of uncoupling protein 1 (Ucp1), and other thermogenic-related genes. Stromal vascular fraction of subcutaneous WAT was extracted for the measurement of type 2 innate lymphocytes (ILC2s) proportions. Results. Glucose tolerance was markedly improved by peripherally administered NMU. Micro-PET/CT suggested that NMU promoted WAT beiging, which was further confirmed by haematoxylin and eosin (H\&E) staining and immunohistochemistry. In diet-induced-obese (DIO) mice, NMU activated thermogenic-related genes in WAT. In addition, NMU stimulated ILC2s in the stromal vascular fraction of WAT. Conclusion. Taken together, our study indicates that peripheral administration of NMU is a potential therapeutic strategy for the promotion of WAT beiging and the improvement of impaired glucose tolerance.
\end{abstract}

\section{Introduction}

Over the past decades, the prevalence of obesity and diabetes increased rapidly, causing great social burden around the world [1-3]. As a major risk factor of type 2 diabetes mellitus, obesity is associated with the imbalance between energy intake and energy expenditure and is characterized with expanded adipose tissue (AT) mass in the body [4, 5]. Classically, AT is divided into three categories, white adipose tissue (WAT), beige adipose tissue, and brown adipose tissue (BAT). The primary function of WAT is energy storage, while beige and brown AT are sites for energy expenditure. BAT consists of multilocular adipocytes with increased numbers of mitochondria that express uncoupling protein 1 (UCP1). BAT dissipates energy through nonshivering thermogenesis, oxidizing fatty acids produced by triglyceride hydrolysis to generate heat. Beige AT refers to brown-like adipocytes sporadically distributed within WAT, which usually arises through the process called WAT beiging upon stimulation of cold, PPAR agonists, or exercise. When stimulated, beige adipocytes express UCP1 protein and exhibit thermogenic capacity at a comparable level to BAT $[4,6,7]$. In obese patients, WAT is excessively accumulated in the body, causing local immune abnormalities, tissue fibrosis, and AT secretome changes, which drive peripheral insulin resistance and contribute to the pathogenesis of type 2 diabetes mellitus [4]. Previous studies have demonstrated that WAT beiging is associated with reduced AT dysfunction and fibrosis. Beige AT is also related to improved glucose and lipid homeostasis [8-10]. However, effective ways to promote WAT beiging are still under investigation. 
Neuromedin U (NMU) is a peptide widely distributed in different kinds of neurons (cholinergic, noncholinergic, and sensory) throughout the body. It is highly expressed in the gastrointestinal tract, as well as many other tissues, including AT $[11,12]$. NMU is conserved across different mammalian species and is involved in multiple important physiological and pathophysiological processes, participating in the regulation of energy homeostasis [12]. In a Denmark population, homozygous carriers of NMU gene mutations had increased prevalence of overweight and obesity [13]. $\mathrm{NMU}^{-/-}$mice were more susceptible to obesity and late-onset hyperglycemia [14]. In rodents, administration of NMU led to not only decreased food intake, lowered body weight, and improved glucose homeostasis, but also activation of BAT, featured by elevated expression of $U c p 1$ in BAT, increased heat production, and body temperature $[15,16]$. Moreover, Ucp1 expression was decreased in BAT of $\mathrm{NMU}^{-1-}$ mice [14]. As a matter of fact, NMU and NMU receptor 1 (NMUR1) were found to express in WAT in human [17]. Nevertheless, whether NMU could promote WAT beiging and improve metabolic homeostasis remains unknown.

Recently, the neuro-immune system crosstalk mediated by NMU-type 2 innate lymphocytes (ILC2s) was discovered. Peripherally injected NMU activated ILC2 cells in small intestine lamina propria and lung [18]. Intraperitoneal injection of an RNase glycoprotein extracted from the Helminth induced ILC2s accumulation in epididymal WAT, leading to weight loss and glucose tolerance improvements in obese mice [19]. In AT, ILC2s, upon activation, produced type 2 cytokines, stimulated eosinophils and type 2 (or M2) macrophages to promote WAT beiging and thermogenesis [20]. However, it remains to be clarified whether peripherally administrated NMU activates ILC2s in AT and facilitates WAT beiging.

In this study, we aimed to investigate the impact of NMU on glucose metabolism and thermogenic AT in diet-induced-obese (DIO) mice. After chronic subcutaneous injection of NMU, blood glucose levels, morphology of AT, and expression of genes related to thermogenesis in BAT and WAT were evaluated. NMU improved glucose tolerance, activated BAT, and promoted WAT beiging in DIO mice. Taken together, our study suggests that peripheral administration of NMU has potential metabolic benefits in promoting WAT beiging and ameliorating impaired glucose tolerance.

\section{Methods}

2.1. Animal Experiments. $\mathrm{C} 57 \mathrm{Bl} / 6$ mice were purchased from Shanghai SLAC Laboratory Animal Co., Ltd, China. Mice were housed in a standard animal room of controlled environment (constant light-dark cycler, lights off at 20:00). Food and water were given ad libitum. 8-week-old mice were randomly divided into normal chow diet (NCD) or high fat diet (HFD) (D12492, Research Diets) groups and fed accordingly until the end of the research. After 6 weeks, mice in the HFD group were randomly divided into normal saline(NS-) treated and NMU-treated (NMU23, Phoenix Pharmaceuticals) group (HFD + NS group, $n=10$; HFD + NMU group, $n=10)$. NMU ( $8 \mu \mathrm{g} / \mathrm{day})$ or NS of the same volume was subcutaneously injected into the interscapular space daily for 14 days. The NCD group was also subcutaneously injected with NS of equal volume (NCD + NS group, $n=12$ ). Body weight and food intake were recorded daily.

\subsection{Intraperitoneal Glucose Tolerance Test (IpGTT).} IpGTT was performed in all three groups after 14 days of NMU or NS injection. Food was withdrawn 12 hours before the test. After measurement of fasting blood glucose using tail blood, mice were treated with $2 \mathrm{~g} / \mathrm{kg} \mathrm{D}$-glucose solution (10\%) by intraperitoneal injection. Blood glucose levels were measured at 15, 30, 60, and 120 min after glucose challenge.

2.3. $18 F-F D G$ Micro-PET/CT. Micro-PET/CT was conducted on a Siemens Inveon PET/CT System in the Department of Nuclear Medicine of Nanjing First Hospital. After 18 hours of fasting and 4 hours of cold exposure $\left(4^{\circ} \mathrm{C}\right)$, mice were kept in $30^{\circ} \mathrm{C}$ environment, with food and water given ad libitum. 30 minutes later, mice were intraperitoneally injected with $18 \mathrm{~F}-\mathrm{FDG}(4 \mu \mathrm{Ci} / \mathrm{g})$. After one hour of radiotracer uptake, mice were anesthetized with constant isoflurane inhalation and fixed in a stretched position for PET/CT scan. CT scanning at $80 \mathrm{kV}$ and $500 \mu \mathrm{A}$ took $10 \mathrm{~min}$, followed by $5 \mathrm{~min}$ of PET signal collection. Coregistration of the reconstructed PET and CT images and all image analysis were performed using the manufacturer's software.

2.4. Isolation of AT and SVF. After 14 days of NMU or NS injection, mice were anesthetized in chambers saturated with isoflurane and then sacrificed by cervical dislocation. For $\mathrm{H} \& \mathrm{E}$ staining and immunohistochemistry, interscapular BAT and inguinal subcutaneous WAT (sWAT) were dissociated and fixed for $24 \mathrm{~h}$ using fat fixative (Wuhan Sevicebio Technology Co., Ltd). For SVF preparation, BAT and sWAT were cut into small pieces $\left(\sim 1 \mathrm{~mm}^{3}\right)$ and digested with $0.1 \%$ type I collagenase (Sigma-Aldrich, USA) in $37^{\circ} \mathrm{C}$ water bath. During digestion, the fat pieces were kept in tubes and mixed every $2 \mathrm{~min}$ by turning the tubes upside down. After 40-45 $\mathrm{min}$, the digestion process was terminated by adding equal volume of phosphate buffer saline (PBS). The specimens were centrifuged at $400 \mathrm{x}$ for $5 \mathrm{~min}$, and the supernatants were then aspirated. The sediments were washed with PBS, filtered through a $100 \mu \mathrm{m}$ nylon mesh, and resuspended in red blood cell lysis buffer (GE, USA). After red blood cell lysis, the remaining SVF was washed again with PBS.

2.5. Histology and Immunofluorescence. BAT and sWAT in fixatives were sectioned after being paraffin-embedded. Sectioned tissues were stained with H\&E for general morphological observation. Immunohistochemistry was conducted using Ucp1 antibodies (BD, Bioscience, 1:100) and secondary antibodies (anti-rabbit, Alexa Fluor 488, Life Technologies Inc., Carlsbad, CA, USA, 1:100). Images were captured and digitalized with microscope (Olympus, Tokyo, Japan). Ucp1 expression was quantified using NIH ImageJ software. 

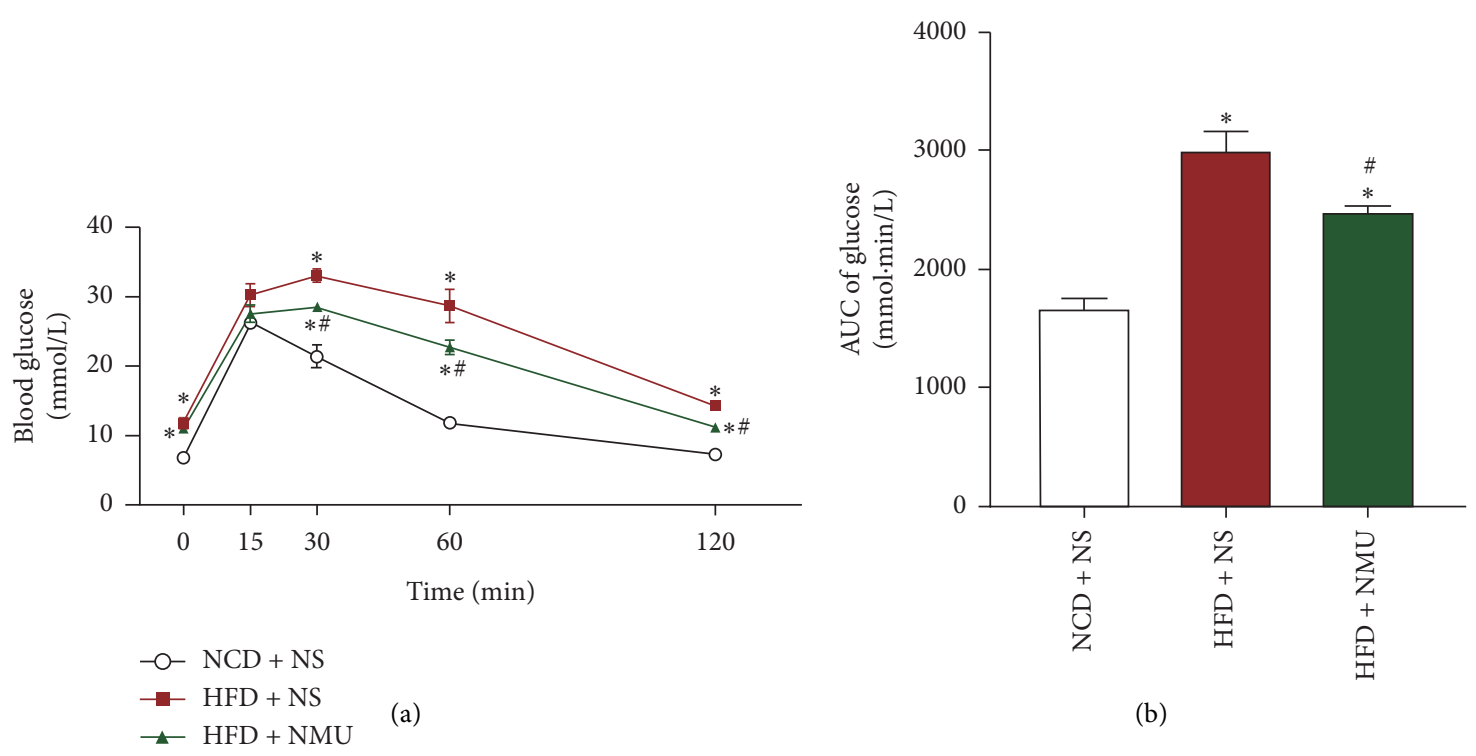

(b)

FIgURE 1: Chronic subcutaneous administration of NMU improves glucose tolerance in DIO mice. (a) Blood glucose levels and (b) area under the curve of glucose levels of ipGTT after NMU administration $(n=5-6) .{ }^{*} p<0.05$ (compared to NCD + NS group). ${ }^{\#} p<0.05$ (compared to HFD + NS group).

2.6. Quantitative Real-Time PCR Analysis ( $q P C R$ ). The total RNA was extracted from BAT and sWAT using trizol (Life Technologies, Thermo Fisher Scientific) following standard protocols. Complementary DNA was prepared from $1 \mathrm{mg}$ RNA with the PrimerScript RT Master Mix (TAKARA, Japan) according to the manufacturer's instructions. Quantitative PCR reactions were run by LightCycler 480 Real-Time PCR System (Roche, Switzerland). Fold changes in gene expression were calculated as $2^{-\triangle \triangle \mathrm{Ct}}$ with $\beta$-actin as the housekeeping gene. Sequences of the primers are listed in Supplementary Table 1.

2.7. Flow Cytometry. Mouse SVF samples were stained with the following antibodies: lineage (Lin) antibodies CD3, CD5, $\mathrm{TCR} \alpha / \beta, \mathrm{CD} 19, \mathrm{CD} 56, \mathrm{CD} 11 \mathrm{c}, \mathrm{CD} 11 \mathrm{~b}$, and CD16 (BD, Bioscience). Other antibodies used for the characterization of ILC2s included L/D, CD45, and ST2 (BD, Bioscience). The stained cells were analyzed on an LSR II Fortessa flow cytometer (BD, Bioscience). ILC2s were identified as $\mathrm{Lin}^{-} \mathrm{CD} 45^{+} \mathrm{ST} 2^{+}$. Data were analyzed using FACSDiva software (BD Bioscience) and FlowJo software version 9.6.4 (Tree Star, Inc).

2.8. Statistical Analyses. All statistical analyses were performed with SPSS version 22.0 (SPSS Inc., USA). Differences were determined using ANOVA tests for normally distributed variables. Data were presented as mean \pm SEM unless otherwise stated. $p$ values $<0.05$ were considered statistically significant.

\section{Results}

3.1. Chronic Subcutaneous Injection of NMU Exerts Beneficial Effects on Glucose Tolerance. Chronic subcutaneous administration of NMU significantly lowered blood glucose levels 30, 60, and 120 minutes after glucose injection
(Figure 1(a)). Area under the curve of blood glucose in ipGTT was also significantly decreased in DIO mice treated with NMU (Figure 1(b)). NMU injection did not induce significant changes in body weight (Supplementary Figure 1(a)) or food intake (Supplementary Figure 1(b)) as compared to NS in DIO mice. Elevated fasting blood glucose (FBG) in DIO mice was not affected by NMU (Supplementary Figure 1(c)). These results suggest that NMU could improve glucose tolerance when energy intake and body weight remain steady.

3.2. NMU Activates BAT and Enhances Thermogenesis. In order to determine whether NMU could increase BAT activity in DIO mice, we performed cold-stimulated microPET/CT on mice of all 3 groups. BAT signal was clearly observed in the control (NCD + NS) group (white circle) but was scarcely present in DIO mice treated with NS. NMU administration activated interscapular BAT as is shown in PET/CT images (Figure 2(a)). H\&E staining showed expanded brown adipocytes with larger lipid droplets in DIO mice, which were reversed in the NMU-treated group (Figures 2(b) and 2(c)). Consistent with phenotypical and morphological changes, immunohistochemistry showed markedly elevated Ucp1 staining in NMU-treated mice (Figure 3(a)). NMU also activated a network of genes that participate in energy dissipating and thermogenesis according to qPCR. The expressions of transcription factors, including $P g c-1 \alpha, E r r \alpha$, and $N r f 1$, were significantly upregulated in NMU-treated DIO mice. NMU also potently increased the expression of several classical BAT markers, such as Ucp1, Cidea, Cox8b, and Isdp5. Components of the mitochondrial electron transport chain and genes related to fatty acid oxidation were also activated (Figure 3(b)). Collectively, our data demonstrates that NMU could restore BAT morphology and function in vivo. 
$\mathrm{NCD}+\mathrm{NS}$

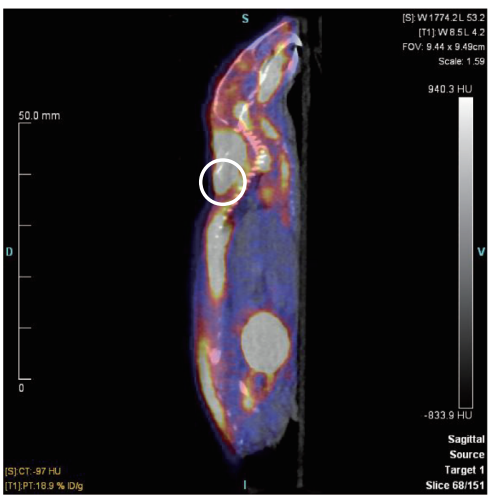

$\mathrm{NCD}+\mathrm{NS}$
$\mathrm{HFD}+\mathrm{NS}$

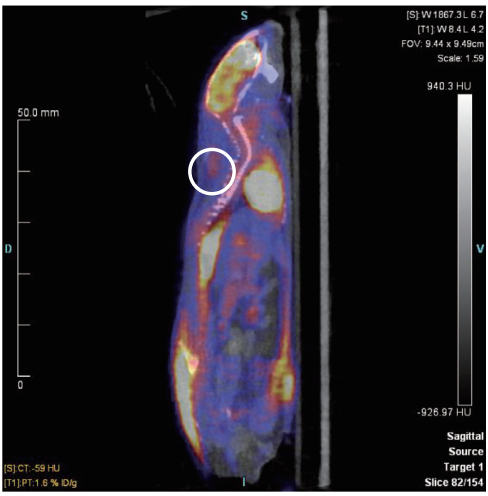

(a)

$\mathrm{HFD}+\mathrm{NS}$

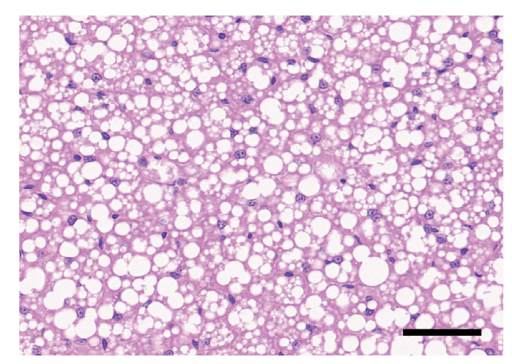

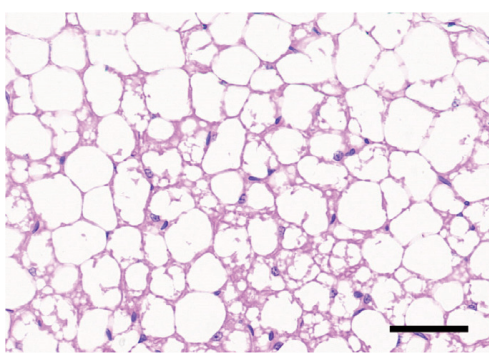

(b)

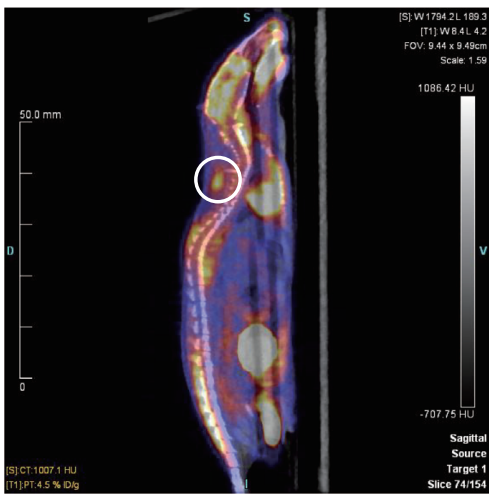

$\mathrm{HFD}+\mathrm{NMU}$

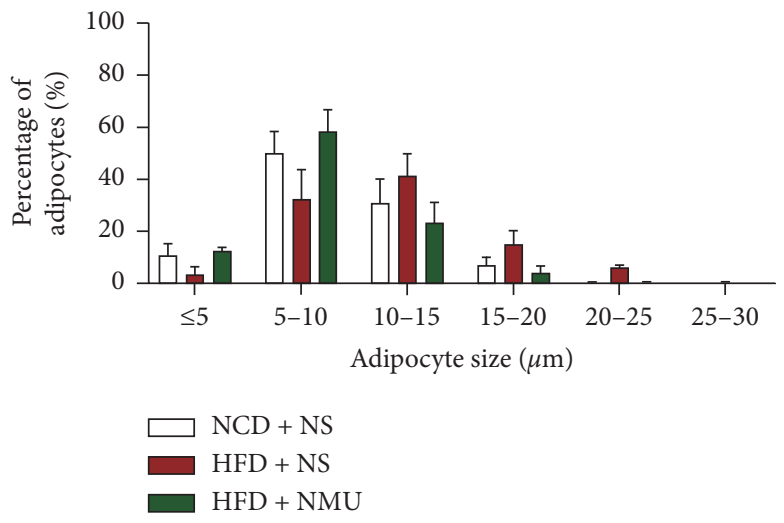

(c)

FIGURE 2: NMU increases FDG-uptake in BAT and restores BAT morphology. (a) Sagittal view of micro-PET/CT image after cold stimulation and 18F-FDG injection. (b) Representative H\&E staining of interscapular BAT. Scale bar, $50 \mu \mathrm{m}$. (c) Quantification of adipocytes of different diameters in interscapular BAT $(n=3) .{ }^{*} p<0.05$ (compared to the NCD + NS group). ${ }^{\#} p<0.05$ (compared to the HFD + NS group).

3.3. NMU Promotes sWAT Beiging. We next evaluated indices related to WAT beiging in the 3 groups. Positive signal of sWAT was detected in the inguinal area by PET/CT. The $\mathrm{PET} / \mathrm{CT}$ signal for inguinal sWAT was negative in DIO mice injected with NS, while after NMU administration, the signal was detected to be higher compared to the other groups (Figure 4(a)). According to H\&E staining of paraffin sections, the apparent adipocyte hypertrophy in DIO mice was ameliorated with NMU treatment (Figures $4(\mathrm{~b})$ and $4(\mathrm{c})$ ). Immunohistochemistry exhibited decreased Ucp1 expression in DIO mice, which was up-regulated by NMU (Figure 5(a) and 5(b)). The expression of several thermogenic-related genes-Nrf1, Ucp1, and Cpt2-were increased by NMU (Figure 4(c)), indicating sWAT beiging induced by subcutaneous NMU treatment.

3.4. NMU Stimulates ILC2s in sWAT. SVF of sWAT was extracted and then analyzed by flow cytometry to examine the changes in ILC2 proportions in different groups. ILC2s were 


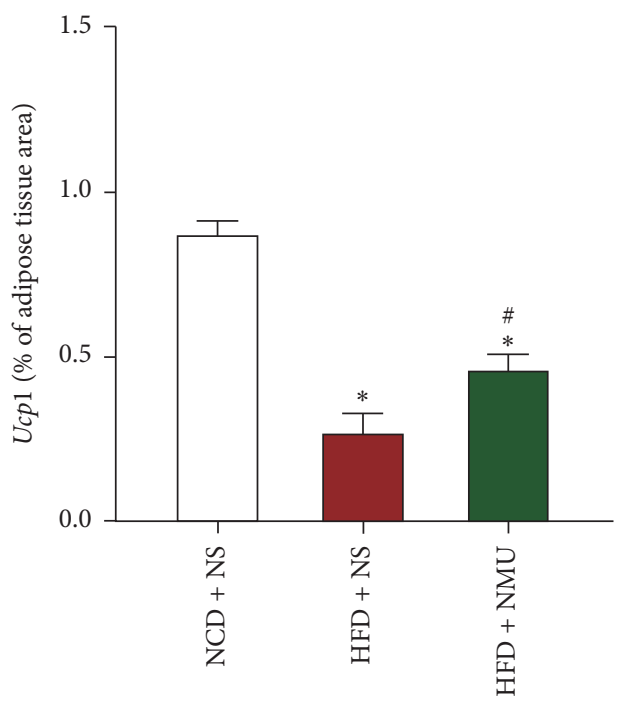

(a)
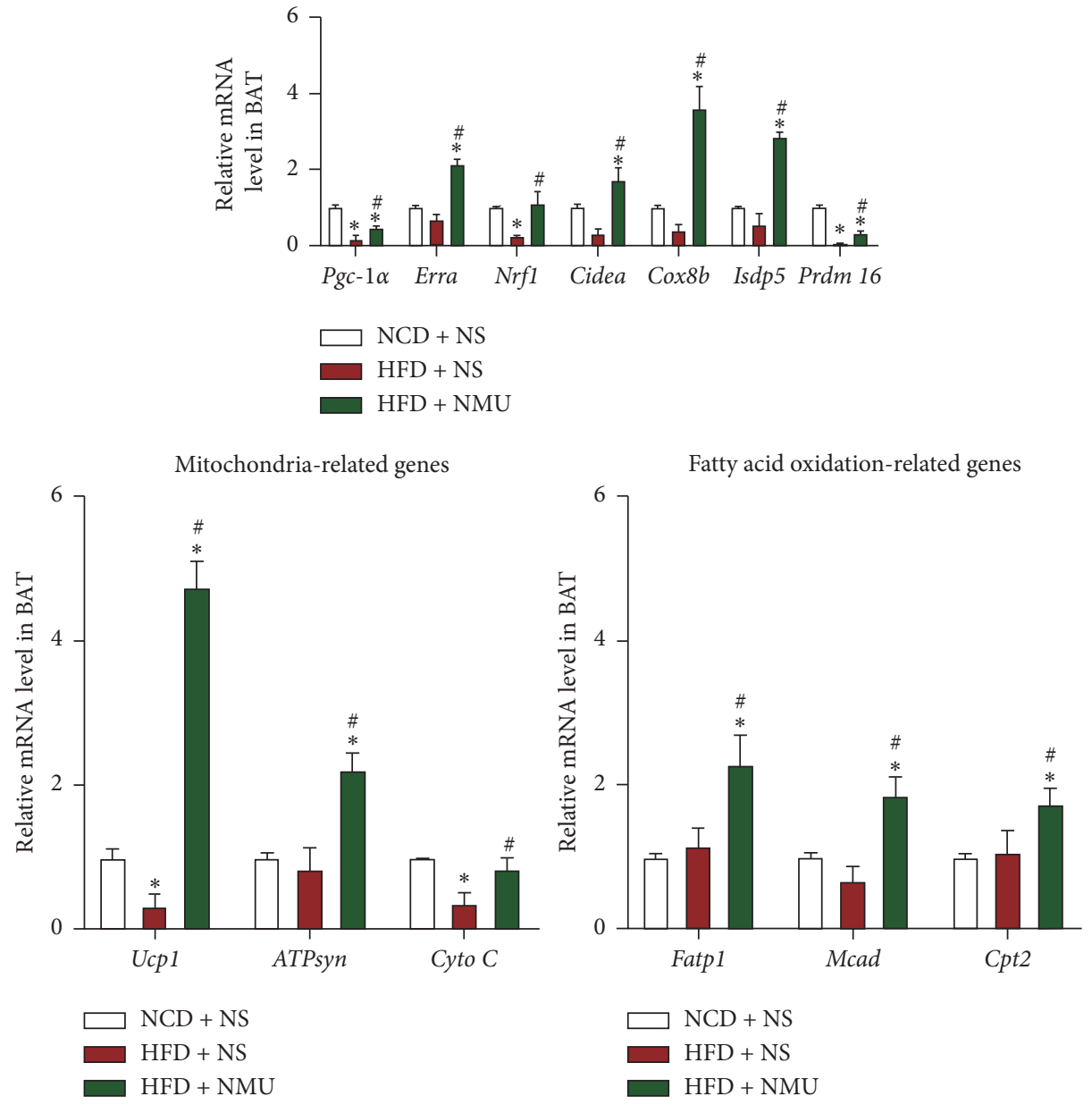

(b)

FIgURe 3: NMU stimulates the expression of thermogenic-related genes in BAT. (a) Quantification of $U c p 1$ stain of BAT ( $n=3$ ). (b) Gene expression profile in BAT $(n=5-6) .{ }^{*} p<0.05$ (compared to the NCD + NS group). ${ }^{\#} p<0.05$ (compared to the HFD + NS group). 
$\mathrm{NCD}+\mathrm{NS}$

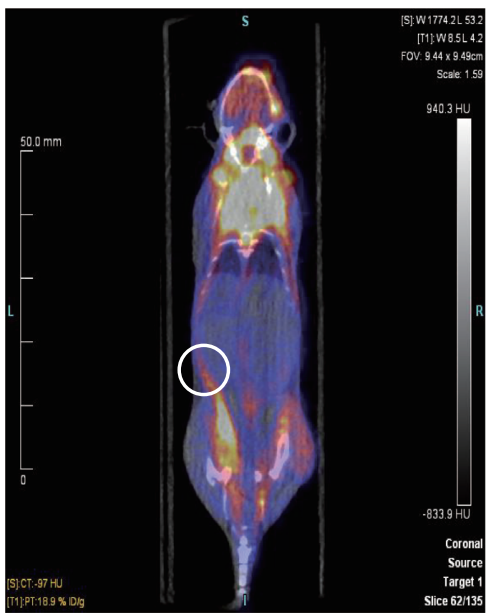

$\mathrm{NCD}+\mathrm{NS}$
$\mathrm{HFD}+\mathrm{NS}$

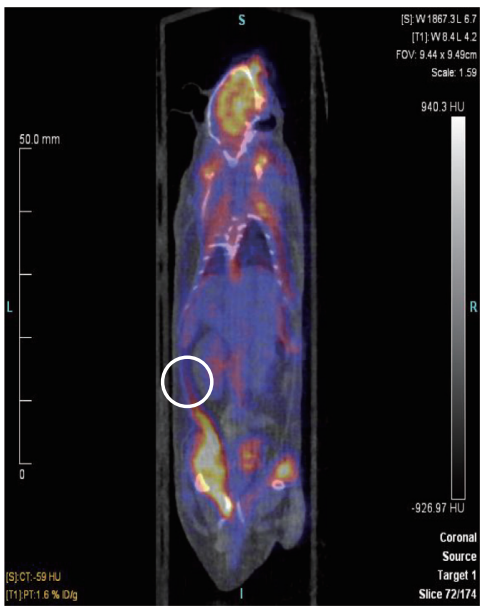

(a)

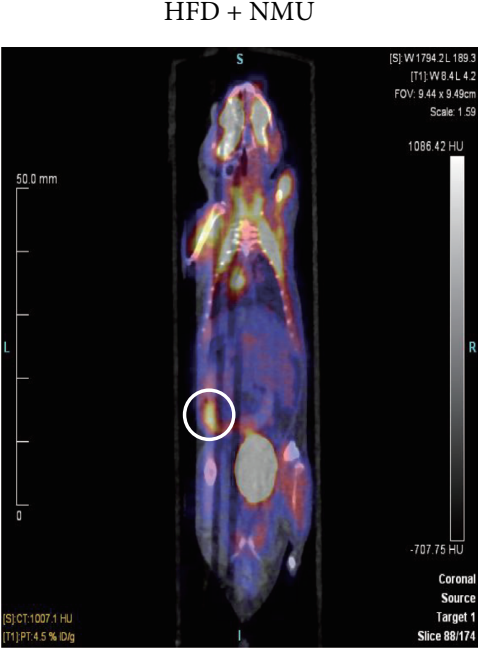

$\mathrm{HFD}+\mathrm{NMU}$

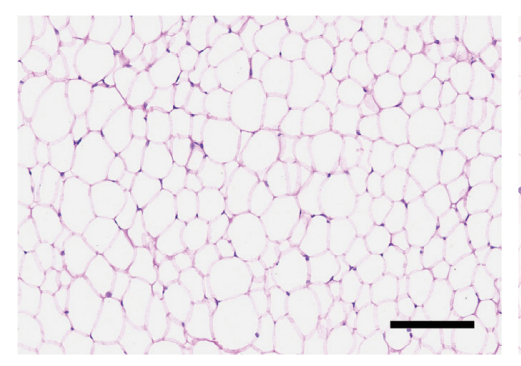

$\mathrm{HFD}+\mathrm{NS}$
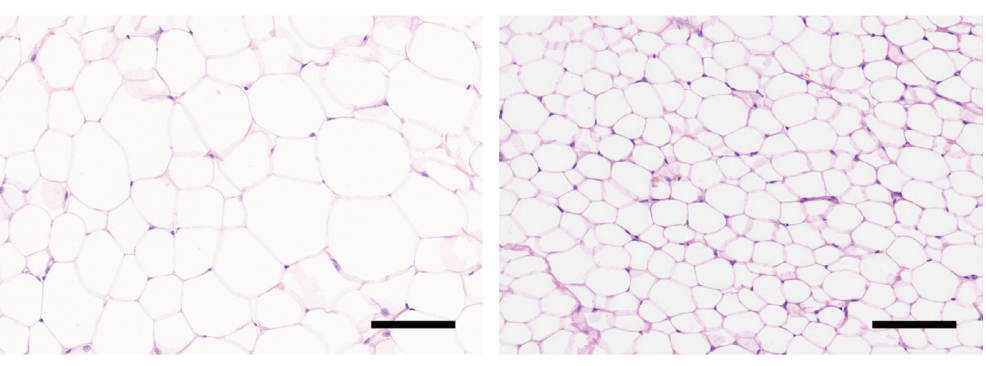

(b)

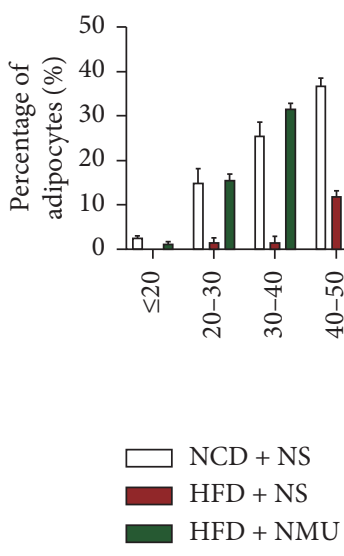

(c)

FIGURE 4: NMU induces brown-like functional and morphological changes in WAT in DIO mice. (a) Coronal view of micro-PET/CT image after cold stimulation and 18F-FDG injection. (b) Representative H\&E staining of sWAT. Scale bar, $100 \mu \mathrm{m}$. (c) Quantification of adipocytes of different diameters in $\operatorname{sWAT}(n=3) .{ }^{*} p<0.05$ (compared to the NCD + NS group). ${ }^{\#} p<0.05$ (compared to the HFD + NS group).

gated on $\mathrm{Lin}^{-} \mathrm{CD} 45^{+} \mathrm{ST} 2^{+}$(Supplementary Figure 1(d)). The percentage of ILC2s in Lin $^{-}$cells is significantly decreased in DIO mice, while the data suggest that in vivo administration of NMU activated ILC2s in sWAT (Figures 6(a) and 6(b)).

\section{Discussion}

Whether NMU enhances thermogenesis in AT and subsequently improves glucose metabolism remain unclear. In this study, we proved that peripherally administered NMU ameliorated impaired glucose tolerance, activated BAT, and promoted WAT beiging. The changes in WAT may be related to ILC2s stimulation.

Since the discovery that NMU modulates energy homeostasis in vivo, studies have been conducted to evaluate the effects of NMU application for the treatment of obesity and diabetes [21]. NMU, NMU analogs, or NMU receptor agonists exerted glucose-lowering effects when applied to rodents via intracerebroventricular or peripheral injections $[16,21-23]$. In our study, the DIO mice were treated with 


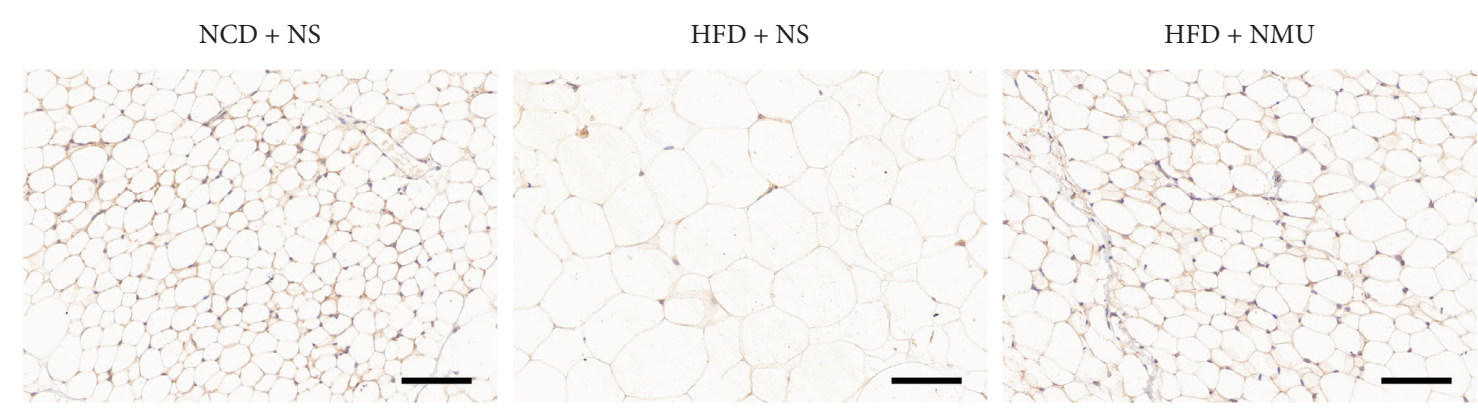

(a)

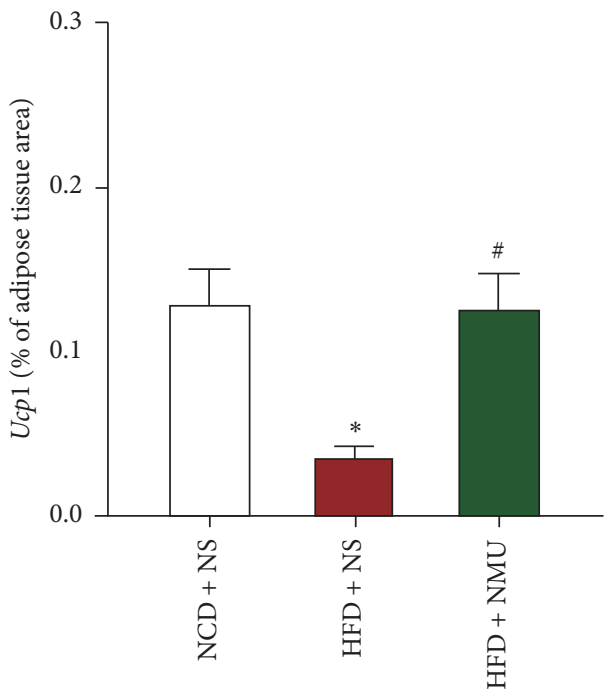

(b)

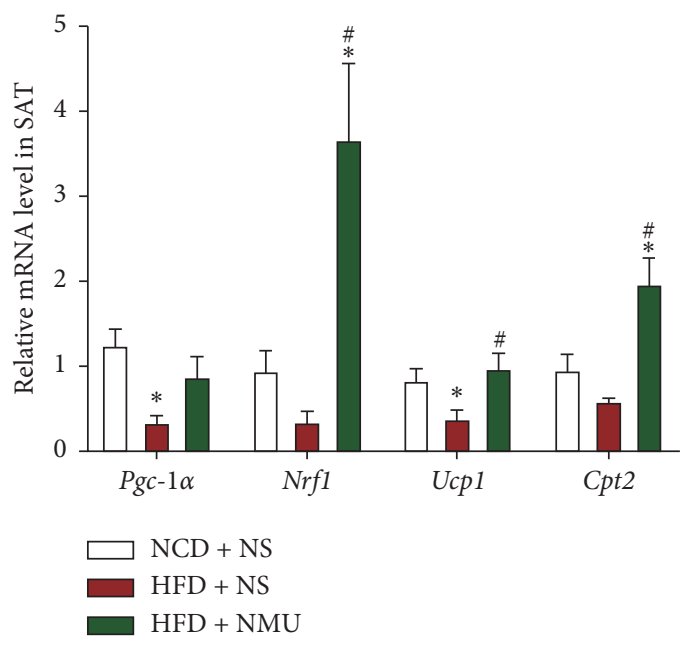

(c)

FigURE 5: NMU activates thermogenic-related gene profile in sWAT. (a) Representative of immunohistochemical images for Ucp1 (brown stain) of inguinal sWAT. Scale bar, $100 \mu \mathrm{m}$. (b) Quantification of Ucp1 stain of sWAT $(n=3)$. (c) Thermogenic-related gene expression profile in sWAT $(n=5-6) .{ }^{*} p<0.05$ (compared to the NCD + NS group). ${ }^{\#} p<0.05$ (compared to the HFD + NS group).
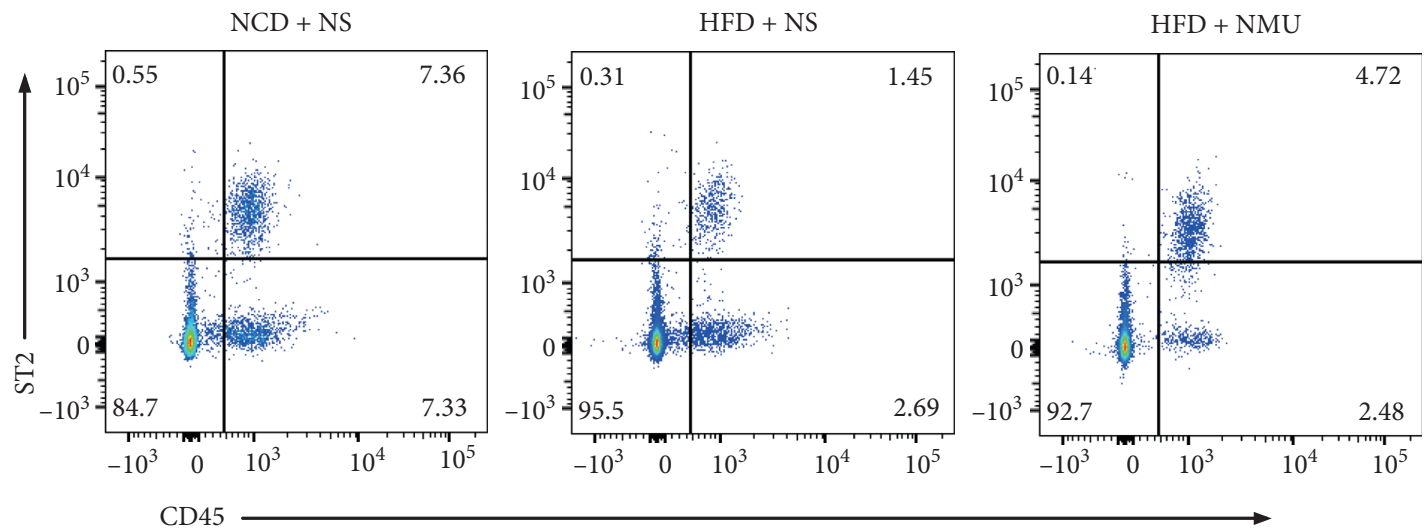

(a)

Figure 6: Continued. 


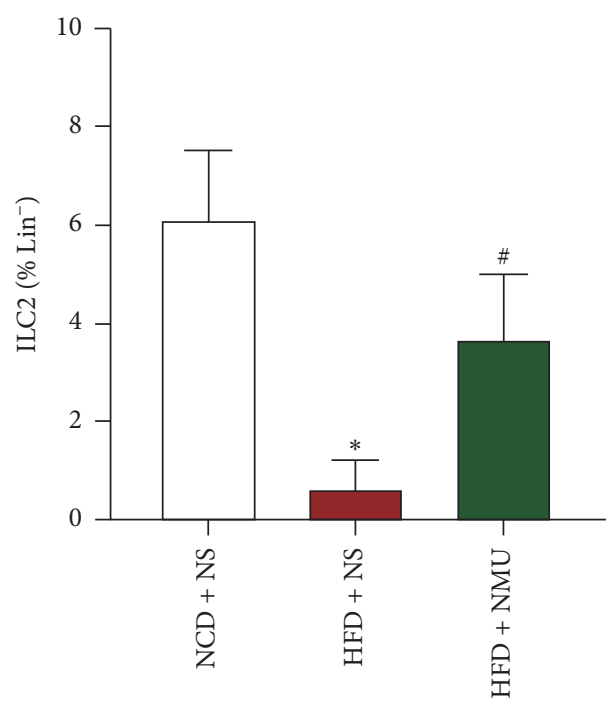

(b)

FIGURE 6: NMU increases ILC2 percentage in WAT in vivo. (a) Representative image of flow cytometry analysis showing the percentage of ILC2s in Lin-cells from mice SVF. (b) Quantification of the percentage of ILC2s in Lin- SVF cells from mice SVF $(n=3-4) .{ }^{*} p<0.05$ (compared to the NCD + NS group). ${ }^{\#} p<0.05$ (compared to the HFD + NS group).

subcutaneously injected NMU. In accordance with previous reports [16], chronic subcutaneous administration of NMU at $8 \mu \mathrm{g} /$ day did not influence body weight, food intake, or fasting blood glucose (Supplementary Figures 1(a)-1(c)). Unexpectedly, glucose tolerance was markedly improved (Figures 1(a) and 1(b)). It was suggested that the main central effects of NMU were anorexia and weight loss [15, 24], indicating that the improved glucose tolerance in our study was not due to central but direct peripheral effect of NMU.

Previous reports have also identified NMU as a potential thermogenic drug. Intracerebroventricular injection of NMU increased the back surface temperature [15] and core temperature [25] in rodents, while the effect was absent in Nmur2-deficient mice [25]. The thermogenic capacity of NMU has been linked to increased BAT activity due to enhanced Ucp1 expression in BAT after NMU administration [15]. Consistent with previous reports, NMU activated BAT and increased BAT thermogenesis in vivo (Figures 2 and 3). Furthermore, we demonstrated that NMU promoted sWAT beiging, characterized by BAT-like morphological, functional, and genetic alterations in sWAT (Figures 4 and 5). To our knowledge, we demonstrate for the first time that chronic peripheral administration of NMU could promote WAT beiging, which may be implicated in the improvement of glucose tolerance. In the obese population, while WAT depots expand, the percentage of adults with detectable BAT decreased in overweight and obesity [26]. Promoting BAT activation and WAT beiging represent attractive therapeutic strategies in combating obesity and insulin resistance $[9,27]$. In adult humans, BAT is a rather vestigial tissue. Less than $10 \%$ of adults had positive scans of BAT by 18 F-FDG PET/ CT. The amount of BAT further diminished with aging and obesity $[26,28]$. Thus, the promotion of WAT beiging may provide more promising therapeutic perspectives among diabetic patients who tend to be obese and aged [9].
Recently, it has been pointed out that immune cells within WAT communicate with local nerve fibers to regulate WAT beiging [29]. ILC2s, identified as non-B, nonT lymphocytes that produce type 2 cytokines, were recognized as the upstream components of the axis of ILC2s, eosinophils, and macrophages, which was involved in the regulation of WAT beiging capacity, local immune homeostasis, and AT health [30-32]. NMU was shown to be expressed by cholinergic neurons in the intestine [11,33]. Intraperitoneal or intranasal-administered NMU activated ILC2s in the small intestine lamina propria and lung $[18,34,35]$. We employed the dosage that was suggested to stimulate ILC2s in vivo [18], with which NMU potently activated ILC2s in sWAT (Figure 6), providing a new perspective in promoting WAT beiging.

In conclusion, we reveal the impact of NMU on glucose metabolism and thermogenesis. Our data also indicate a potential role of the neuro-immune regulation mediated by NMU and ILC2s in promoting WAT beiging. These findings demonstrate the beneficial metabolic effects of peripherally administered NMU, identifying NMU as a potential drug to enhance thermogenesis and improve glucose tolerance.

\section{Data Availability}

All data generated or analyzed during this study are included in this article and its supplementary information files.

\section{Ethical Approval}

This study was performed in line with the principles of the Declaration of Helsinki. Approval was granted by the Research Animal Care Committee of Affiliated Drum Tower Hospital, Medical School of Nanjing University, Nanjing, China (2019AE01006). 


\section{Conflicts of Interest}

The authors declare that they have no conflicts of interest.

\section{Authors' Contributions}

Yue Yuan, Hongdong Wang, and Jielei He contributed equally to this work and are regarded as co-first authors. Y. Y., H. D. W., and J. L. H. conceptualized the study; H. D. W. and Y. B. were responsible for methodology; J. L. H., H. D. W., and Y. Y. were responsible for formal analysis; Y. Y., J. L. H., and H. X. S. were responsible for data curation; Y. Y. prepared the original draft; H. D. W. and Y. B. reviewed and edited the manuscript; Y. B. supervised the study; Y. B. and D. L. Z. acquired funding.

\section{Acknowledgments}

This work was supported by the National Natural Science Foundation of China Grant Awards (81770819, 82030026, $81970689,82070837,81970704,81703294,81800752$, $81900787,82000735,82000775$, and 81800719), the National Key Research and Development Program of China (2016YFC1304804 and 2017YFC1309605), the Jiangsu Provincial Key Medical Discipline (ZDXKB2016012), the Key Project of Nanjing Clinical Medical Science, the Jiangsu Provincial Medical Talent (ZDRCA2016062), the Six Talent Peaks Project of Jiangsu Province of China (YY-086), the Scientific Research Project of the Fifth Phase of "333 Project" of Jiangsu Province of China, the Doctor of Entrepreneurship and Innovation Program of Jiangsu Province, and the Fundamental Research Funds for the Central Universities (021414380444).

\section{Supplementary Materials}

Supplementary Figure 1 Chronic subcutaneous injection of NMU did not impact body weight (a), $(n=10-12)$, food intake (b), $(n=3)$ or FBG (c), $(n=5-6)$. (d) Gating strategy for ILC2s in flow cytometric analysis. Lin: CD3, CD5, TCR $\alpha /$ $\beta, \mathrm{CD} 19, \mathrm{CD} 56, \mathrm{Fc \varepsilon R} \alpha, \mathrm{CD} 11 \mathrm{c}, \mathrm{CD} 11 \mathrm{~b}, \mathrm{CD} 16 .{ }^{*} p<0.05$ (compared to NCD + NS group). ${ }^{\#} p<0.05$ (compared to HFD + NS group). Supplementary Table 1 Primers Used for Quantitative real-time PCR analysis. (Supplementary Materials)

\section{References}

[1] CDC, Maps of Diabetes and Obesity, by County, CDC, Atlanta, GA, USA, 2004, 2010, and 2016, https://www.cdc.gov/ diabetes/data/center/slides.html.

[2] N. H. Cho, J. E. Shaw, S. Karuranga et al., "IDF Diabetes Atlas: global estimates of diabetes prevalence for 2017 and projections for 2045," Diabetes Research and Clinical Practice, vol. 138, pp. 271-281, 2018.

[3] WHO, Global Report on Diabetes, WHO, Geneva, Switzerland, 2016.

[4] C. R. Kahn, G. Wang, and K. Y. Lee, "Altered adipose tissue and adipocyte function in the pathogenesis of metabolic syndrome," Journal of Clinical Investigation, vol. 129, no. 10, pp. 3990-4000, 2019.
[5] F. Bragg, K. Tang, Y. Guo et al., "Associations of general and central adiposity with incident diabetes in Chinese men and women," Diabetes Care, vol. 41, no. 3, pp. 494-502, 2018.

[6] K. Ikeda, P. Maretich, and S. Kajimura, "The common and distinct features of Brown and beige adipocytes," Trends in Endocrinology \& Metabolism, vol. 29, no. 3, pp. 191-200, 2018.

[7] L. Sidossis and S. Kajimura, "Brown and beige fat in humans: thermogenic adipocytes that control energy and glucose homeostasis," Journal of Clinical Investigation, vol. 125, no. 2, pp. 478-486, 2015.

[8] S. Y. Min, J. Kady, M. Nam et al., "Human "brite/beige" adipocytes develop from capillary networks, and their implantation improves metabolic homeostasis in mice," Nature Medicine, vol. 22, no. 3, pp. 312-318, 2016.

[9] B. S. Finlin, H. Memetimin, B. Zhu et al., "The $\beta 3$-adrenergic receptor agonist mirabegron improves glucose homeostasis in obese humans," Journal of Clinical Investigation, vol. 130, no. 5, pp. 2319-2331, 2020.

[10] Y. Chen, K. Ikeda, T. Yoneshiro et al., "Thermal stress induces glycolytic beige fat formation via a myogenic state," Nature, vol. 565, no. 7738, pp. 180-185, 2019.

[11] J. Ballesta, F. Carlei, A. E. Bishop et al., "Occurrence and developmental pattern of neuromedin U-immunoreactive nerves in the gastrointestinal tract and brain of the rat," Neuroscience, vol. 25, no. 3, pp. 797-816, 1988.

[12] V. G. Martinez and L. O’Driscoll, "Neuromedin U: a multifunctional neuropeptide with pleiotropic roles," Clinical Chemistry, vol. 61, no. 3, pp. 471-482, 2015.

[13] I. Hainerová, S. S. Torekov, J. Ek et al., "Association between neuromedin U gene variants and overweight and obesity," The Journal of Clinical Endocrinology \& Metabolism, vol. 91, no. 12, pp. 5057-5063, 2006.

[14] R. Hanada, H. Teranishi, J. T. Pearson et al., "Neuromedin U has a novel anorexigenic effect independent of the leptin signaling pathway," Nature Medicine, vol. 10, no. 10, pp. 1067-1073, 2004.

[15] K. Nakahara, A. Akagi, S. Shimizu et al., "Involvement of endogenous neuromedin $\mathrm{U}$ and neuromedin $\mathrm{S}$ in thermoregulation," Biochemical and Biophysical Research Communications, vol. 470, no. 4, pp. 930-935, 2016.

[16] A. M. Peier, K. Desai, J. Hubert et al., "Effects of peripherally administered neuromedin $U$ on energy and glucose homeostasis," Endocrinology, vol. 152, no. 7, pp. 2644-2654, 2011.

[17] P. G. Szekeres, A. I. Muir, L. D. Spinage et al., "Neuromedin U is a potent agonist at the orphan $\mathrm{G}$ protein-coupled receptor FM3," Journal of Biological Chemistry, vol. 275, no. 27, pp. 20247-20250, 2000.

[18] V. Cardoso, J. Chesné, H. Ribeiro et al., "Neuronal regulation of type 2 innate lymphoid cells via neuromedin U," Nature, vol. 549, no. 7671, pp. 277-281, 2017.

[19] E. Hams, R. Bermingham, F. A. Wurlod et al., "The helminth T2 RNase $\omega 1$ promotes metabolic homeostasis in an IL-33and group 2 innate lymphoid cell-dependent mechanism," The FASEB Journal, vol. 30, no. 2, pp. 824-835, 2016.

[20] M. Uhm and A. R. Saltiel, "White, brown, and beige; type 2 immunity gets hot," Immunity, vol. 42, no. 1, pp. 15-17, 2015.

[21] A. D. Prins, A. V. Eeckhaut, I. Smolders, D. Tourwe, and S. Ballet, "Neuromedin U and structural analogs: an overview of their structure, function and selectivity," Current Medicinal Chemistry, vol. 27, no. 39, pp. 6744-6768, 2020.

[22] P. Ingallinella, A. M. Peier, A. Pocai et al., "PEGylation of Neuromedin $U$ yields a promising candidate for the treatment 
of obesity and diabetes," Bioorganic \& Medicinal Chemistry, vol. 20, no. 15, pp. 4751-4759, 2012.

[23] H. Nagai, T. Kaisho, K. Yokoyama et al., "Differential effects of selective agonists of neuromedin $\mathrm{U} 1$ and $\mathrm{U} 2$ receptors in obese and diabetic mice," British Journal of Pharmacology, vol. 175 , no. 2, pp. 359-373, 2018.

[24] M. Nakazato, R. Hanada, N. Murakami et al., "Central effects of neuromedin $U$ in the regulation of energy homeostasis," Biochemical and Biophysical Research Communications, vol. 277, no. 1, pp. 191-194, 2000.

[25] A. Peier, J. Kosinski, K. Cox-York et al., "The antiobesity effects of centrally administered neuromedin $U$ and neuromedin $\mathrm{S}$ are mediated predominantly by the neuromedin $\mathrm{U}$ receptor 2 (NMUR2)," Endocrinology, vol. 150, no. 7, pp. 3101-3109, 2009.

[26] A. M. Cypess, S. Lehman, G. Williams et al., "Identification and importance of brown adipose tissue in adult humans," New England Journal of Medicine, vol. 360, no. 15, pp. 1509-1517, 2009.

[27] M. Chondronikola, E. Volpi, E. Børsheim et al., "Brown adipose tissue improves whole-body glucose homeostasis and insulin sensitivity in humans," Diabetes, vol. 63, no. 12, pp. 4089-4099, 2014.

[28] J. Orava, P. Nuutila, T. Noponen et al., "Blunted metabolic responses to cold and insulin stimulation in brown adipose tissue of obese humans," Obesity, vol. 21, no. 11, pp. 2279-2287, 2013.

[29] A. Guilherme, F. Henriques, A. H. Bedard, and M. P. Czech, "Molecular pathways linking adipose innervation to insulin action in obesity and diabetes mellitus," Nature Reviews Endocrinology, vol. 15, no. 4, pp. 207-225, 2019.

[30] W. R. Bolus and A. H. Hasty, "Contributions of innate type 2 inflammation to adipose function," Journal of Lipid Research, vol. 60, no. 10, pp. 1698-1709, 2019.

[31] H. Maazi and O. Akbari, "Type two innate lymphoid cells: the Janus cells in health and disease," Immunological Reviews, vol. 278, no. 1, pp. 192-206, 2017.

[32] A. B. Molofsky, J. C. Nussbaum, H.-E. Liang et al., "Innate lymphoid type 2 cells sustain visceral adipose tissue eosinophils and alternatively activated macrophages," Journal of Experimental Medicine, vol. 210, no. 3, pp. 535-549, 2013.

[33] M. Honzawa, T. Sudoh, N. Minamino, K. Kangawa, and H. Matsuo, "Neuromedin U-like immunoreactivity in rat intestine: regional distribution and immunohistochemical study," Neuropeptides, vol. 15, no. 1, pp. 1-9, 1990.

[34] C. S. N. Klose, T. Mahlakõiv, J. B. Moeller et al., "The neuropeptide neuromedin $\mathrm{U}$ stimulates innate lymphoid cells and type 2 inflammation," Nature, vol. 549, no. 7671, pp. 282-286, 2017.

[35] A. Wallrapp, S. J. Riesenfeld, P. R. Burkett et al., "The neuropeptide NMU amplifies ILC2-driven allergic lung inflammation," Nature, vol. 549, no. 7672, pp. 351-356, 2017. 